UCT-TP-232/96

June 1996

\title{
Pions at Finite Temperature from QCD Sum Rules
}

\author{
C. A. Dominguez ${ }^{a}$, M. S. Fetea ${ }^{a}$, and M. Loewe ${ }^{b}$ \\ a Institute of Theoretical Physics and Astrophysics, University of Cape Town, \\ Rondebosch 7700, South Africa \\ $b \quad$ Facultad de Fisica, Pontificia Universidad Catolica de Chile, \\ Casilla 306, Santiago 22, Chile
}

\begin{abstract}
The temperature corrections to the current algebra Gell-Mann, Oakes, and Renner $(\mathrm{GMOR})$ relation in $S U(2) \otimes S U(2)$ are investigated in the framework of QCD sum rules. There are no corrections at leading order in the quark masses. At the next to leading order we find corrections of the form $m_{q}^{2} T^{2}$, which are small except near the critical temperature. As a by-product we obtain the temperature behaviour of the pion mass, which is essentially constant, except near the critical temperature where it increases with $T$.
\end{abstract}


Due to its small mass, the pion plays a special role in the dynamics of hot hadronic matter. Therefore, it is quite important to understand the temperature behaviour of the pion's Green function. The pion mass $\mu_{\pi}(T)$ has been studied in a variety of frameworks, such as Chiral Perturbation Theory (low temperature expansion) [1], the Linear Sigma Model [2], the Mean Field Approximation [3], the Virial Expansion [4], etc. There seems to be a reasonable consensus that $\mu_{\pi}(T)$ is essentially independent of $T$, except possibly near the critical temperature $T_{c}$ where $\mu_{\pi}(T)$ increases with $T$. While the pion is (hadronically) stable at $T=0$, it is expected to develop a width (imaginary part of the Green function) at non-zero temperature, such width being interpreted as a damping coefficient which should diverge at the critical temperature for deconfinement. This follows from a proposal [5] - [6] (see also [7]) to consider the width of a hadron as a phenomenological order parameter for the deconfinement phase transition. In fact, as the temperature is increased and the hadron melts, its width should increase until it becomes infinite at $T=T_{c}$, thus ensuring that no resonance peaks remain in the hadronic spectral function. The latter should become a smooth function of the energy and coincide with its perturbative QCD value. These properties have been confirmed by detailed calculations in the framework of the virial expansion [8] and the Linear Sigma Model [9].

In this paper we study the temperature corrections to the well known Gell-Mann, Oakes, and Renner (GMOR) relation of current algebra [10]

$$
f_{\pi}^{2} \mu_{\pi}^{2}=-\left(m_{u}+m_{d}\right)<\bar{q} q>
$$

where $f_{\pi} \simeq 93 \mathrm{MeV}$, and $<\bar{u} u>\simeq<\bar{d} d>\equiv<\bar{q} q>\simeq-0.01 \mathrm{GeV}^{3}$. We do this in the framework of QCD sum rules at finite temperature [5], [11], [12]. As a byproduct of our analysis, we obtain the temperature dependence of the pion mass. 
We begin by considering the following two-point function (at $T=0$ )

$$
\Pi_{5 \mu}(q)=i \int d^{4} x e^{i q \cdot x}<0\left|T\left(A_{\mu}(x) j_{5}^{\dagger}(0)\right)\right| 0>=i q_{\mu} \Pi_{5}\left(q^{2}\right),
$$

where $A_{\mu}(x)=: \bar{d}(x) \gamma_{\mu} \gamma_{5} u(x):$, and $j_{5}(x)=: \bar{d}(x) i \gamma_{5} u(x):$ Calculating $\Pi_{5}\left(q^{2}\right)$ in perturbative QCD to order $\mathcal{O}\left(m_{q}^{3}\right)$, and incorporating the leading non-perturbative corrections, we find

$$
\begin{aligned}
\left.\Pi_{5}\left(q^{2}\right)\right|_{\mathrm{QCD}}= & -\frac{3}{8 \pi^{2}}\left(m_{u}+m_{d}\right) \ln \left(-q^{2} / \nu^{2}\right)+\frac{3}{4 \pi^{2}} m_{u} m_{d}\left(m_{u}+m_{d}\right) \frac{1}{q^{2}} \\
& +\frac{2<\bar{q} q>}{q^{2}}+\frac{1}{2}\left(m_{u}+m_{d}\right)^{2} \frac{<\bar{q} q}{q^{4}}-\frac{1}{8} \frac{\left(m_{u}+m_{d}\right)}{q^{4}}<\frac{\alpha_{s}}{\pi} G^{2}>.
\end{aligned}
$$

Using $m_{u} \simeq 5 \mathrm{MeV}, m_{d} \simeq 10 \mathrm{MeV}$ [13], and $<\frac{\alpha_{s}}{\pi} G^{2}>\simeq(1-4) \times 10^{-2} \mathrm{GeV}^{4}$ [14], the above expression can be safely approximated for $-q^{2} \simeq 1 \mathrm{GeV}^{2}$ as

$$
\begin{aligned}
\left.\Pi_{5}\left(q^{2}\right)\right|_{\mathrm{QCD}} \simeq & \frac{-3}{8 \pi^{2}}\left(m_{u}+m_{d}\right) \ln \left(-q^{2} / \nu^{2}\right) \\
& +\frac{2<\bar{q} q>}{q^{2}}-\frac{1}{8} \frac{\left(m_{u}+m_{d}\right)}{q^{4}}<\frac{\alpha_{s}}{\pi} G^{2}>.
\end{aligned}
$$

On the hadronic side, saturation with the lowest hadronic state (the pion) yields

$$
\left.\Pi_{5}\left(q^{2}\right)\right|_{\mathrm{HAD}}=\frac{2 f_{\pi}^{2} \mu_{\pi}^{2}}{\left(m_{u}+m_{d}\right)} \frac{1}{\mu_{\pi}^{2}-q^{2}} .
$$

We shall ignore in the sequel contributions from higher resonances $\left(\pi^{\prime}, \pi^{\prime \prime}\right.$, etc.) because the analysis will be basically restricted to energies below $1 \mathrm{GeV}$, in which case these hadronic contributions are absorbed into the continuum.

Invoking Cauchy's theorem in the form

$$
\left.\frac{1}{\pi} \int_{0}^{s_{0}} s^{N} \operatorname{Im} \Pi_{5}(s)\right|_{\mathrm{HAD}} d s=-\left.\frac{1}{2 \pi i} \int_{C\left(\left|s_{0}\right|\right)} s^{N} \Pi_{5}(s)\right|_{\mathrm{QCD}} d s,
$$

where $N=0,1,2 \cdots$, leads to Finite Energy Sum Rules (FESR). The first two FESR in our case read

$$
2 f_{\pi}^{2} \mu_{\pi}^{2}=-2\left(m_{u}+m_{d}\right)<\bar{q} q>+\frac{3}{8 \pi^{2}}\left(m_{u}+m_{d}\right)^{2} \int_{0}^{s_{0}} d s
$$




$$
2 f_{\pi}^{2} \mu_{\pi}^{4}=\frac{1}{8}\left(m_{u}+m_{d}\right)^{2}<\frac{\alpha_{s}}{\pi} G^{2}>+\frac{3}{8 \pi^{2}}\left(m_{u}+m_{d}\right)^{2} \int_{0}^{s_{0}} s d s,
$$

where $s_{0}$ is the continuum threshold. Equation (7) becomes the GMOR relation at leading order in the quark masses. We have left the explicit form of the trivial integrals above, in order to compare later with the FESR at finite temperature, where these integrals cannot be calculated analytically in closed form.

Next, we reconsider the above FESR at finite temperature. Thermal corrections to $\left.\Pi_{5}\left(q^{2}\right)\right|_{\text {QCD }}$ can be calculated in the standard fashion [5], [11, [12, and we find for the imaginary part

$$
\begin{aligned}
\left.\operatorname{Im} \Pi_{5}(s, T)\right|_{\mathrm{QCD}}= & \frac{3}{8 \pi}\left(m_{u}+m_{d}\right)\left[1-2 n_{F}\left(\frac{\sqrt{s}}{2 T}\right)\right] \\
& +\frac{\pi}{2}\left(m_{u}+m_{d}\right) T^{2} \delta(s),
\end{aligned}
$$

where $n_{F}(x)=\left(1+e^{x}\right)^{-1}$ is the Fermi thermal factor. In addition, the non-perturbative vacuum condensates will develop a temperature dependence. For $<\bar{q} q>_{T}$ we shall use the results of [3] away from the chiral limit, i.e. for $m_{q} \neq 0$. The gluon condensate is basically independent of $T$, except very close to the critical temperature $T_{c}$ [15], so that we shall take it as a constant. On the hadronic side, the pion mass and decay constant will develop a temperature dependence, and so will $s_{0}$. The latter follows from the notion that as the resonance peaks in the spectral function become broader, the onset of the continuum should shift towards threshold [5], [16]. The temperature behaviour of this asymptotic freedom threshold can be obtained from the lowest dimension FESR associated to the two-point function involving the axial-vector currents [5], [16], provided $f_{\pi}(T)$ is known, viz.

$$
\begin{aligned}
8 \pi^{2} f_{\pi}^{2}(T)= & \frac{1}{2} \int_{0}^{s_{0}(T)} d z^{2} v(z)\left[3-v^{2}(z)\right]\left[1-2 n_{F}\left(\frac{z}{2 T}\right)\right] \\
& +\int_{0}^{\infty} d z^{2} v(z)\left[3-v^{2}(z)\right] n_{F}\left(\frac{z}{2 T}\right)
\end{aligned}
$$

where $v(z)=\left(1-\left(m_{u}+m_{d}\right)^{2} / z^{2}\right)^{\frac{1}{2}}$. We have used $f_{\pi}(T)$ as determined in [3] (for $m_{q} \neq 0$ ), and solved the above FESR for $s_{0}(T)$. The result is shown in Fig.1, together 
with the input $f_{\pi}(T)$, as well as $<\bar{q} q>_{T}$, both from [3]. It is interesting to notice that for temperatures not too close to $T_{c}$, say $T<0.8 T_{c}$, the following scaling relation holds to a good approximation

$$
\frac{f_{\pi}^{2}(T)}{f_{\pi}^{2}(0)} \simeq \frac{<\bar{q} q>_{T}}{<\bar{q} q>} \simeq \frac{s_{0}(T)}{s_{0}(0)}
$$

The FESR, Eqs.(7) and (8) now become

$$
\begin{aligned}
G(T) & \equiv 2 f_{\pi}^{2}(T) \mu_{\pi}^{2}(T)+2\left(m_{u}+m_{d}\right)<\bar{q} q>_{T} \\
& =\frac{3}{8 \pi^{2}}\left(m_{u}+m_{d}\right)^{2}\left\{\frac{4 \pi^{2}}{3} T^{2}+\int_{0}^{s_{0}(T)} d s\left[1-2 n_{F}\left(\frac{\sqrt{s}}{2 T}\right)\right]\right\} \\
2 f_{\pi}^{2}(T) \mu_{\pi}^{4}(T) & =\frac{3}{8 \pi^{2}}\left(m_{u}+m_{d}\right)^{2}\left\{\frac{\pi}{3}<\alpha_{s} G^{2}>+\int_{0}^{s_{0}(T)}\left[1-2 n_{F}\left(\frac{\sqrt{s}}{2 T}\right)\right] s d s\right\} .
\end{aligned}
$$

In Fig. 2 we show the ratio

$$
\frac{G(T)}{G(0)}=\frac{4 \pi^{2}}{3} \frac{T^{2}}{s_{0}(0)}+\frac{1}{s_{0}(0)} \int_{0}^{s_{0}(T)} d s\left[1-2 n_{F}\left(\frac{\sqrt{s}}{2 T}\right)\right]
$$

for $s_{0}(0)=1 \mathrm{GeV}^{2}$ (reasonable changes around this value have basically no influence on the results). Qualitatively, our result is in agreement with expectations: since both $f_{\pi}(T)$ and $<\bar{q} q>_{T}$ decrease with $T$, the same should be true of $G(T)$. From the point of view of the sum rule, Eq.(14), the decrease of $G(T)$ is due to the decrease of $s_{0}(T)$ (notice that $T^{2} / s_{0}(0) \ll 1$ in the temperature range under consideration). Quantitatively, it would be interesting to compare our result with that of the low temperature expansion, once this becomes available (to determine the $\mathcal{O}\left(T^{2}\right)$ correction one needs the two-loop, $\mathcal{O}\left(p^{6}\right)$, calculation of the parameters entering $G(T)[17)$.

Using $f_{\pi}(T)$ and $<\bar{q} q>_{T}$ as input, plus $s_{0}(T)$ obtained from Eq.(10), it is possible to use the two FESR above, Eqs.(12)-(13), to obtain two independent determinations of $\mu_{\pi}(T)$. The results of this calculation, shown in Fig.(3), are in good agreement with each other, 
as well as with results from other methods [1]- 团. This is rather important, as it provides strong support for the validity of the QCD sum rule program at finite temperature.

Acknowledgements The work of (CAD) and (MSF) has been supported in part by the FRD (South Africa), and that of (ML) by Fondecyt (Chile) under grant No.1950797.

\section{References}

[1] J. Gasser and H. Leutwyler, Phys. Lett. B 184 (1987) 83.

[2] A. Larsen, Z. Phys. C, Particles \& Fields, 33 (1986) 291; C. Contreras and M. Loewe, Int. J. Mod. Phys. A 5 (1990) 2297.

[3] A. Barducci, R. Casalbuoni, S. de Curtis, R. Gatto, and G. Pettini, Phys. Rev. D 46 (1992) 2203.

[4] A. Schenk, Nucl. Phys. B363 (1991) 97; Phys. Rev. D47 (1993) 5138.

[5] C. A. Dominguez and M. Loewe, Phys. Lett. B 233 (1989) 201.

[6] C. A. Dominguez, Nucl. Phys. B (Proc. Suppl.) 15 (1990) 225; C. A. Dominguez and M. Loewe, Nucl. Phys. B (Proc. Suppl.)16 (1990) 403; Z. Phys. C, Particles \& Fields, 49 (1991) 423.

[7] R. D. Pisarski, Phys. Lett. B 110 (1982) 155.

[8] H. Leutwyler and A. V. Smilga, Nucl. Phys. B 342 (1990) 302.

[9] C. A. Dominguez, M. Loewe, and J. C. Rojas, Z. Phys. C, Particles \& Fields, 59 (1993) 63.

[10] M. Gell-Mann, R. Oakes, and B. Renner, Phys. Rev. 175 (1968) 2195. 
[11] A. I. Bochkarev and M. E. Shaposnikov, Nucl. Phys. B 268 (1986) 220.

[12] C. A. Dominguez and M. Loewe, Z. Phys. C, Particles \& Fields, 51 (1991) 69; ibid. 58 (1993) 273; Phys. Rev. D 52 (1995) 3143.

[13] C. A. Dominguez and E. de Rafael, Ann. Phys. (N.Y.) 174 (1987) 372.

[14] R. A. Bertlmann et al., Z. Phys. C, Particles \& Fields, 39 (1988) 231; C.A. Dominguez and J. Solà, ibid 40 (1988) 63.

[15] P. Gerber and H. Leutwyler, Nucl. Phys. B321 (1989) 387.

[16] A. Barducci, R. Casalbuoni, S. de Curtis, R. Gatto, and G. Pettini, Phys. Lett. B244 (1990) 311.

[17] H. Leutwyler and D. Toublan, private communication and work in progress.

\section{Figure Captions}

Figure 1: The ratios $f_{\pi}^{2}(T) / f_{\pi}^{2}(0)$, Solid curve (a); $\left\langle\bar{q} q>_{T} /<\bar{q} q>\right.$, dotted curve (b), both from [3]; and $s_{0}(T) / s_{0}(0)$, dashed curve (c), from the FESR Eq. (10).

Figure 2: The ratio of the GMOR relation, Eq.(14).

Figure 3: The ratio $\mu_{\pi}(T) / \mu_{\pi}(0)$ obtained by solving the FESR Eq.(12), curve (a), and Eq.(13), curve (b). In both cases, $f_{\pi}(T)$ and $<\bar{q} q>_{T}$ from [3] were used as input. 